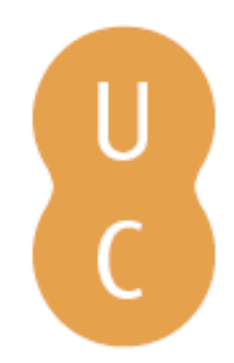

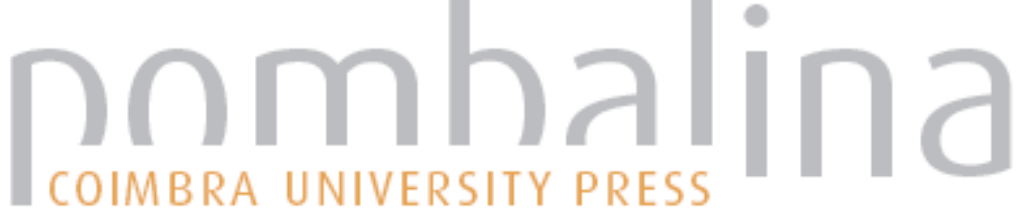

\section{A estrutura óssea do fémur}

Autor(es): $\quad$ Trabucho, L.

Publicado por: Imprensa da Universidade de Coimbra; Gradiva

URL persistente:

URI:http://hdl.handle.net/10316.2/32625

DOI:

DOI:http://dx.doi.org/10.14195/978-989-26-0485-5_14

Accessed : $\quad$ 26-Apr-2023 13:13:48

A navegação consulta e descarregamento dos títulos inseridos nas Bibliotecas Digitais UC Digitalis, UC Pombalina e UC Impactum, pressupõem a aceitação plena e sem reservas dos Termos e Condições de Uso destas Bibliotecas Digitais, disponíveis em https://digitalis.uc.pt/pt-pt/termos.

Conforme exposto nos referidos Termos e Condições de Uso, o descarregamento de títulos de acesso restrito requer uma licença válida de autorização devendo o utilizador aceder ao(s) documento(s) a partir de um endereço de IP da instituição detentora da supramencionada licença.

Ao utilizador é apenas permitido o descarregamento para uso pessoal, pelo que o emprego do(s) título(s) descarregado(s) para outro fim, designadamente comercial, carece de autorização do respetivo autor ou editor da obra.

Na medida em que todas as obras da UC Digitalis se encontram protegidas pelo Código do Direito de Autor e Direitos Conexos e demais legislação aplicável, toda a cópia, parcial ou total, deste documento, nos casos em que é legalmente admitida, deverá conter ou fazer-se acompanhar por este aviso. 
(Página deixada propositadamente em branco) 
MARIA PAULA SERRA DE OLIVEIRA

Coordenadora

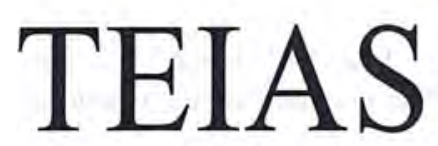

MATEMÁTICAS

Frentes na Ciência e na Sociedade 
(C) Gradiva - Publicações, L. da / Imprensa da Universidade de Coimbra, 2004 Coordenação editorial: Maria Paula Serra de Oliveira

Tradução: Artur Soares Alves

Carlota Isabel Leitão Pires Simões

Francisco José Craveiro de Carvalho

João Filipe Cortez Rodrigues Queiró

José Miguel Dordio Martinho de Almeida Urbano

Lia Sandra dos Santos

Mário da Silva Rosa

Paulo Eduardo Aragão Aleixo Neves de Oliveira

Revisão do texto: Isabel Pedrome

Capa: António Barros [Imprensa da Universidade. Coimbra], com imagem de

E. M. de Melo e Castro, "Fract 010 explod MC", Dezembro de 2003

[Fractal original gerado no Fractint com tratamento no Photoshop 7.0]

Infografia: Estúdios Estímulus [design]

Paginação: António Resende e Victor Hugo Fernandes

Impressão e acabamento: G.C. - Gráfica de Coimbra, L. da

Reservados os direitos para Portugal por:

Gradiva - Publicações, L. ${ }^{\text {da }}$ e Imprensa da Universidade de Coimbra

Gradiva - Publicações, L. ${ }^{d a}$

Rua Almeida e Sousa, 21, r/c, esq. • 1399-041 Lisboa

Telefs. $213974067 / 8 \cdot 213971357 \cdot 213953470$

Fax $213953471 \cdot$ Email: gradiva@ip.pt

URL: http://www.gradiva.pt

Imprensa da Universidade de Coimbra

Rua Antero de Quental, 195 • 3000-033 Coimbra

Telefs. 351239853110

Fax 3512398531 19 e-mail: fjrpress@ci.uc.pt

URL: http://www.imp.uc.pt

ISBN: 972-662-970-5

1." edição: Maio de 2004

Depósito legal n. ${ }^{\circ} 210431 / 04$

OBRA PUBLICADA COM O PATROCÍNIO DE:

CENTRO DE MATEMÁTICA DA UNIVERSIDADE DE COIMBRA DEPARTAMENTO DE MATEMÁTICA DA UNIVERSIDADE DE COIMBRA

FCT Fundação para a Ciência e a Tecnologia

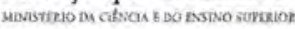




\section{Trabucho}

Departamento de Matemática

da Faculdade de Ciências

da Universidade de Lisboa

\section{A estrutura óssea do fémur}

\section{RESUMo}

O esqueleto dos vertebrados é essencialmente constituido por dois tipos de estrutura óssea: osso cortical - denso e compacto; osso trabecular - poroso e esponjoso.

Em 1892, o físiologista alemão Julius Wolff propôs uma explicação para a distribuição destes dois tipos de estrutura designada, actualmente, por lei de Wolff.

A ideia subjacente consiste numa visão dinâmica da estrutura óssea como consequência da sua adaptabilidade às diversas solicitações externas. Num local onde as tensões mecânicas passem a ser mais elevadas existirá deposição de matéria óssea, enquanto num outro, onde a partir de determinado momento as tensões diminuam substancialmente passará a existir absorção de matéria óssea. A este processo de absorção/deposição de matéria óssea dá-se o nome de remodelação óssea.

Possuir um modelo fiável de remodelação óssea é da maior importância no caso dos implantes ortopédicos, do tratamento de fracturas, da biomecânica desportiva e da prevenção da osteoporose, do tratamento de assimetrias ósseas durante o crescimento, etc.

A lei de Wolff afirma ainda que, perante uma mudança de estímulos exteriores, a remodelação se dá segundo direcções privilegiadas associadas às direcções de maior tensão mecânica. Esta afirmação tem conduzido à elaboração dos mais variados modelos analíticos e empíricos nos últimos cem anos. 
Com o advento dos grandes meios de computação e o desenvolvimento de conceitos matemáticos associados à optimização de estruturas foi possível começar a ter uma maior compreensão do processo mecânico de remodelação óssea e, simultaneamente, generalizar a maioria dos modelos propostos neste último século. Nestes novos modelos, a lei de Wolff surge, naturalmente, associada a condições necessárias de estacionaridade de determinados funcionais de energia.

A discretização destes modelos matemáticos tem conduzido a simulações numéricas que, além de permitirem uma melhor compreensão do fenómeno da remodelação óssea, começam a desempenhar uma enorme ajuda na prática clínica.

Neste artigo ilustrar-se-ão estes conceitos aplicando-os ao estudo da estrutura óssea do fémur.

\section{Optimização de estruturas. Caso unidimensional}

Considere-se uma barra ocupando o intervalo $] 0, L[\subset R$ de secção de área $A$ fixa num dos extremos, por exemplo em $x=0$, e solicitada por uma força aplicada $F$, no outro extremo, localizado em $x=L$.

Considere-se que o material de que a barra é feita possa possuir uma microestrutura formada pela combinação de dois materiais-base, homógeneos e isótropos, de módulo de elasticidade $E^{+}$e $E^{-}$, nas proporções $\gamma$ e $(I-\gamma)$, respectivamente, com

$$
0 \leq \gamma \leq 1
$$

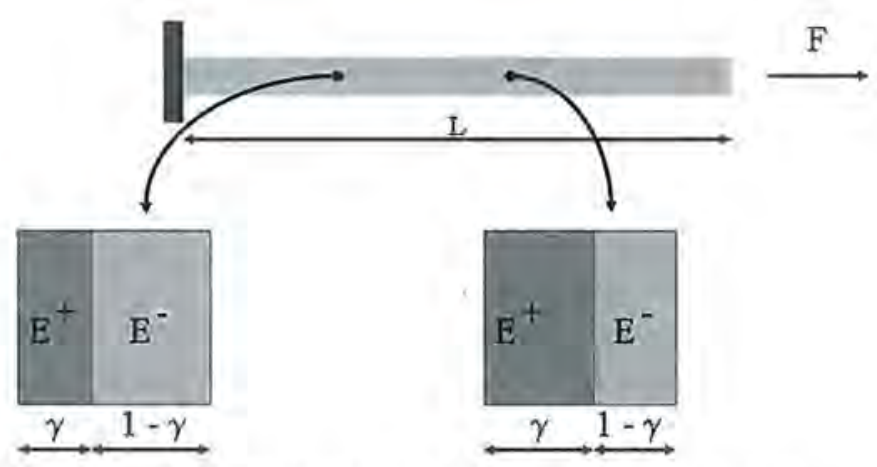

Fig. 1 - Exemplificação do tipo de microestrutura da barra 
A massa específica de cada um destes materiais será designada por $\rho^{+}$e $\rho^{-}$, respectivamente. Por simplicidade consideram-se estes dois materiais bem ordenados, isto é:

$$
E^{+}>E^{-}, \rho^{+}>\rho^{-} \text {. }
$$

Nos problemas de optimização de topologia é usual considerar a minimização do trabalho das forças aplicadas, isto é, calcular a distribuição da fracção volúmica $\gamma$, de modo a que a grandeza $F u(L)$ seja o menor possível, onde $u$ designa deslocamento axial da barra ( $c f$. Bendsøe, M. P. e Kikuchi, N., 1988 e Bendsøe, M. P., 1995). Sem mais restrições, a solução para este problema é dada por $\gamma=1$. Consequentemente, toda a barra seria constituída pelo material mais forte, $E^{+}$. Porém, se houver um custo em adicionar material $E^{+}$, poderá acontecer que este seja proibitivo e que, em algumas partes, ou no seu todo, a barra tenha de ser constituída tảmbém por material $E^{-}$, numa proporção a determinar. Além disso, não há razão para esperar que o valor de $\gamma$ numa determinada secção seja o mesmo que noutra secção, isto é, poderá ter-se $\gamma=\gamma(x)$.

Considere-se então o problema de minimizar o funcional

$$
l(\gamma)=F u(L)+k \int_{0}^{L} \rho^{H} A,
$$

onde as unidades da constante $k$ são trabalho/massa representando, fisicamente, o trabalho necessário realizar para adicionar ao sistema uma unidade de massa. A grandeza $\rho^{H}$ representa a densidade macroscópica (homogeneizada) da barra formada pelos dois materiais em questão e será uma função de $\gamma$.

Para que a estrutura esteja em equilibrio, o campo de deslocamentos $u$ tem de verificar as equações de equilíbrio, que escreveremos na forma variacional, isto $\dot{e}, u \in V=\left\{v \in H^{1}(0, L): v(0)=0\right\}$ :

$$
\int_{0}^{L} E^{H} A u^{\prime} v^{\prime} d x-F v(L)=0, \quad \forall v \in V,
$$

onde $E^{H}$ representa o módulo de elasticidade macroscópico (homogeneizado) do material de que é feita a barra e o símbolo «'» designam a derivada em ordem à variável $x$.

A utilização da teoria da homogeneização é uma das várias possibilidades para representar a influência da microestrutura nas equações de equilíbrio, obtidas a partir da mecânica dos meios contínuos. Trata-se de uma das teorias mais apropriadas a uma análise matemática do problema (cf. Bendsøe, M. P., e Kikuchi, N., 1988; Bendsøe, M. P., 1995; Guedes, J. M., e Kikuchi, N., 1990; e Murat, F., e Tartar, L., 1997). 
Resumindo, o problema de optimização de topologia é constituído pela minimização do funcional (3), sujeito às equações (1) e (4) e, no caso presente, para materiais verificando (2). Para resolver este problema pode considerar-se o lagrangiano:

$$
\begin{aligned}
L=F u(L) & +k \int_{0}^{L} \rho^{H} A d x+\lambda\left[\int_{0}^{L} E^{H} A u v d x-F v(L)\right] d x+ \\
& +\int_{0}^{L} \tau^{+}(\gamma-1) d x+\int_{0}^{L} \tau^{-} \gamma d x
\end{aligned}
$$

onde $\lambda, \tau^{+}$e $\tau^{-}$são multiplicadores de Lagrange associados aos constrangimentos (4), $\gamma \leq 1$ e $\gamma \geq 0$, respectivamente.

Das condições necessárias de estacionaridade do lagrangiano $L$ obtém-se, para além de (4) e para $x \in] 0, L[$ :

$$
\begin{aligned}
& v=-\frac{1}{\lambda} u, \\
& k \frac{d p^{H}}{d \gamma} A-\frac{d E^{H}}{d \gamma} A|u|^{2}+\tau^{+}-\tau^{-}=0 \\
& \tau^{+} \geq 0, \\
& \tau^{-} \geq 0, \\
& \tau^{+}(\gamma-1)=0 \\
& \tau^{-} \gamma=0
\end{aligned}
$$

Até este momento não se especificou o tipo de microestrutura da barra. Considere-se, sem perda de generalidade, o caso mais simples de uma microestrutura laminada, formada pelos materiais $E^{+}$e $E^{-}$orientados segundo um sistema de eixos paralelo a $O x_{1}$ e a $O x_{2}$, nas proporções $\gamma$ e (1- $\gamma$ ), respectivamente (cf. figura 1 ).

Da teoria da homogeneização tem-se ( $c f$. Bendsøe, M. P., 1995; Guedes, J. M., e Kikuchi, N., 1990; e Murat, F., e Tartar, L., 1997):

$$
p^{H}=\gamma \rho^{+}+(1-\gamma) \rho^{-}, \frac{1}{E^{H}}=\frac{\gamma}{E^{+}}+\frac{1-\gamma}{E^{-}},
$$

Assim, a equação (7) toma a forma:

$$
k\left(\rho^{+}-\rho^{-}\right) A-\frac{E^{+} E^{-}\left(E^{+}-E^{-}\right)}{\left[\gamma E^{-}+(1-\gamma) E^{+}\right]^{2}}\left|u^{\prime}\right|^{2} A+\tau^{+}-\tau^{-}=0 .
$$


Da equação de equilíbrio obtém-se $\left(E^{H} A u^{\prime}\right)^{2}=F^{2}$, o que, substituindo na equação anterior, conduz a

$$
\underbrace{k\left(\rho^{+}-\rho^{-}\right) A-F^{2} \frac{\left(E^{+}-E^{-}\right)}{A E^{+} E^{-}}}_{x}=-\tau^{+}+\tau^{-}
$$

Juntamente com (8)-(11), esta é a equação que conduz à solução do problema. De facto, se o custo $(k)$ for muito baixo, tem-se $\chi<0$ e, consequentemente, $\tau=0, \tau^{+}>0$, o que implica, de (10), que $\gamma=1$. Por outras palavras, se o custo de adicionar material for muito baixo, a barra será formada pelo material mais rígido (geralmente mais oneroso), de módulo de elasticidade $E^{+}$. Se o custo de adicionar material for elevado, tem-se $\chi>0 \mathrm{e}$, consequentemente, $\tau>0, \tau^{+}=0$, o que implica, de (11), que $\gamma=0$. Isto é, a barra será formada pelo material mais fraco, de módulo de elasticidade $E^{-}$.

Resumindo, tem-se:

$$
\begin{aligned}
& \chi>0 \Rightarrow \tau^{+}=0, \tau^{-}>0 \Rightarrow \gamma=0 \Rightarrow E^{H}=E^{-} \\
& \chi<0 \Rightarrow \tau^{+}>0, \tau^{-}=0 \Rightarrow \gamma=1 \Rightarrow E^{H}=E^{+} \\
& \chi=0 \Rightarrow \tau^{+}=0, \tau^{-}=0 \Rightarrow 0 \leq \gamma \leq 1 \Rightarrow E^{H}=\frac{1}{\frac{\gamma}{E^{+}}+\frac{1-\gamma}{E^{-}}}
\end{aligned}
$$

ou, de forma equivalente:

$$
\begin{aligned}
& F^{2}<k\left(\rho^{+}-\rho^{-}\right) A^{2} \frac{E^{+} E^{-}}{E^{+}-E^{-}} \Rightarrow \gamma=0 \Rightarrow E^{H}=E^{-}, \\
& F^{2}>k\left(\rho^{+}-\rho^{-}\right) A^{2} \frac{E^{+} E^{-}}{E^{+}-E^{-}} \Rightarrow \gamma=1 \Rightarrow E^{H}=E^{+}, \\
& F^{2}=k\left(\rho^{+}-\rho^{-}\right) A^{2} \frac{E^{+} E^{-}}{E^{+}-E^{-}} \Rightarrow 0 \leq \gamma \leq 1 \Rightarrow E^{H}=\frac{1}{\frac{\gamma}{E^{+}}+\frac{1-\gamma}{E^{-}}},
\end{aligned}
$$

Verifica-se assim que, quando a força aplicada é tal que

$$
F^{2}=k\left(\rho^{+}-\rho^{-}\right) A^{2} \frac{E^{+} E^{-}}{E^{+}-E^{-}},
$$

a solução não é única, podendo a barra ser formada por qualquer combinação dos dois materiais. 
Em conclusão, à medida que se aumenta a força, mantendo o custo (k) fixo, (ou à medida que se diminui o custo mantendo a força $F$ fixa), a solução do problema é dada por uma barra constituída pelo material mais fraco de propriedades $\left(E^{-}, \rho^{-}\right)$até que se atinja a relação (19), onde existirá uma multiplicidade de soluções caracterizada por $0 \leq \gamma \leq 1$, para se passar a ter uma barra constituida apenas pelo material de propriedades $\left(E^{+}, \rho^{+}\right)$quando a força aumentar ainda mais.

De um modo empírico, pode dizer-se que à medidà que as solicítações exteriores variam, o material de que a barra é feita se adapta para suportar as novas solicitações. É este mesmo tipo de análise, envolvendo a minimização do trabalho das forças aplicadas juntamente com o custo de adicionar material e utilizando a teoria da homogeneização para representar a influência da microestrutura nas equações de equilibrio, obtidas através da mecânica dos meios contínuos, que nos dará uma compreensão da estrutura óssea do fémur.

\section{A Estrutura óssea do fémur}

A procura de modelos capazes de reproduzir e explicar, de um ponto de vista mecânico, o processo de remodelação óssea começou com o trabalho pioneiro do fisiologista alemão Julius Wolff. De um ponto de vista estrutural, o osso é extremamente complexo. No fémur podem distinguir-se essencialmente três zonas: uma formada por osso cortical, denso e compacto (cerca de $85 \%$ da matéria óssea); outra caracterizada pela ausência de material ósseo e onde se inserem vários sistemas e tecidos biológicos que não contribuem, de forma substancial, para a resistência mecânica do fémur e uma terceira zona formada por material esponjoso e poroso designada por zona trabecular ( $c f$. figura 2).

Em alguns pontos desta região trabecular poderá o leitor observar que a orientação das trabéculas parece ser bem determinada. Foi esta observação que permitiu a Julius Wolff elaborar o que ficou conhecido como lei de Wolff e que afirma que, perante uma mudança de estímulos exteriores, a remodelação se dá segundo direcções privilegiadas associadas às direcções de maior tensão mecânica (cf. figuras 2 e 3 ).

Durante o século XX vários foram os modelos que surgiram para explicar os fenómenos de remodelação óssea. Alguns baseiam-se em factos experimentais, outros em considerações empiricas, mas quase todos consistem numa equação diferencial ordinária cuja variável independente é a fracção volúmica (ou a massa específica) e a variável dependente o tempo. Porém, a completa compreensão e descrição do fenómeno da remodelação óssea é ainda um problema em aberto. 


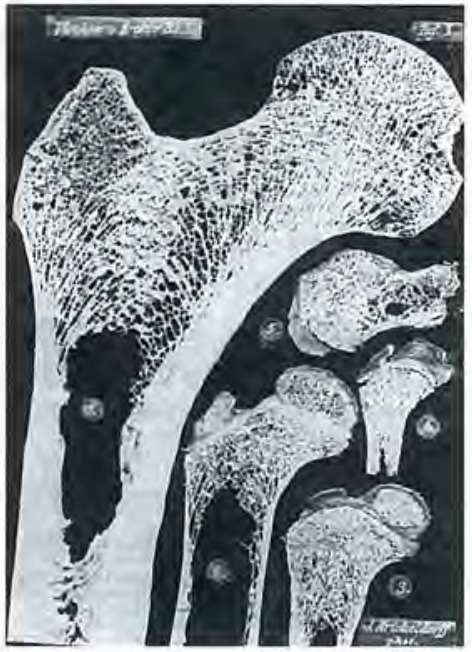

Fig. 2 - Fotografia da estrutura do fèmur apresentada no artigo de J. Wolff de 1870

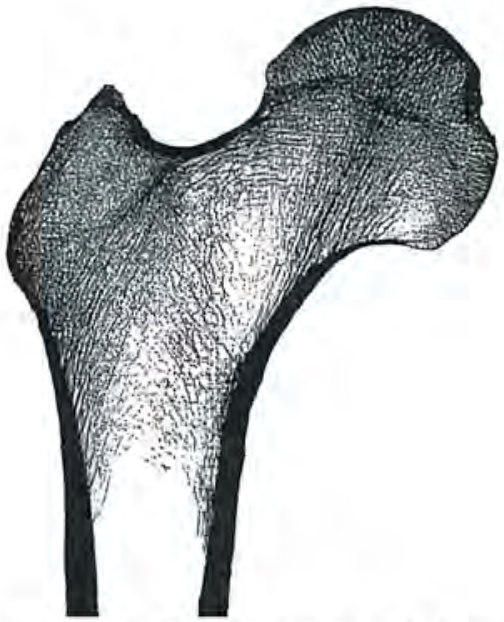

Fig. 3 - Radiografia da cabeça do fémur

Nos últimos anos pensou-se que, do ponto de vista macroscópico, o fenómeno de remodelação óssea poderia ser visto como um processo de optimização de uma estrutura. O funcional a minimizar seria o trabalho 
das forças aplicadas, com um termo de penalização para a adição de massa, e as equações de estado seriam as equações de equilíbrio fornecidas pela teoria da elasticidade linearizada. A influência da microestrutura seria modelada utilizando a teoria da homogeneização. Isto é, trata-se de uma generalização directa, ao caso tridimensional, do anteriormente exposto.

Mais especificamente, seja $\Omega$ um subconjunto de $R^{3}$, aberto, limitado, conexo. Seja $x=\left(x_{i}\right), 1 \leq i \leq 3$, um ponto genérico de $\Omega$. Seja $\partial \Omega=\overline{\Gamma_{0}} \cup \overline{\Gamma_{1}}$ com $\overline{\Gamma_{0}} \cap \overline{\Gamma_{1}}=\phi$ sua fronteira. Considere-se um corpo que ocupa o volume $\Omega$ e está submetido a um sistema de forças interiores, por unidade de volume, dadas por $f=\left(f_{i}(x)\right), 1 \leq i \leq 3$; e a um sistema de forças de superficie, $F=\left(F_{i}(x)\right), 1 \leq i \leq 3$, por unidade de área, em $\Gamma_{1}$. Designe $n=\left(n_{i}\right), 1 \leq i \leq 3$, a normal unitária exterior a $\partial \Omega$ e $u=\left(u_{i}\right), 1 \leq i \leq 3$, o campo de deslocamentos. É possível demonstrar que, neste caso, o estado de tensão num ponto $x \in \bar{\Omega}$ fica completamente caracterizado pelo tensor das tensões de Cauchy, $\sigma(u)=\left(\sigma_{i j}(u)\right), 1 \leq i, j \leq 3$; o estado de extensão num ponto $x \in \bar{\Omega}$ fica completamente caracterizado pelo tensor das extensões linearizado, $e(u)=\left(e_{i j}(u)\right), 1 \leq i, j \leq 3$, onde:

$$
e_{i j}(u)=\frac{1}{2}\left(\frac{\partial u_{j}}{\partial x_{j}}+\frac{\partial u_{j}}{\partial x_{i}}\right) \text {, para todo } 1 \leq i, j \leq 3 .
$$

Neste caso a lei de Hooke (lei constitutiva) toma a forma geral:

$$
\sigma_{i j}(u)=a_{i j k m} e_{k m}(u),
$$

onde $a_{i j k m}=\left(a_{i j k m}(x)\right)$ designam as componentes de um tensor de quarta ordem e onde se utilizou a convenção de soma no índice repetido.

Considerando que na parte da fronteira $\Gamma_{0}$ o corpo está fixo, a teoria da elasticidade linearizada diz-nos que o equilíbrio do corpo é regido pelo sistema seguinte:

$$
\begin{gathered}
-\partial_{j} \sigma_{i j}=f_{i}, \text { em } \Omega, \\
u_{i}=0, \text { sobre } \Gamma_{1}, \\
\sigma_{i j} n_{j}=F_{i}, \text { sobre } \Gamma_{2},
\end{gathered}
$$

onde $\partial_{j}$ designa a derivada parcial em relação à variável $x_{j}$ e onde, novamente, se utilizou a convenção de soma no índice repetido. Trata-se de um sistema de três equações diferenciais com derivadas parciais, do 
tipo eliptico, para as três componentes do campo de deslocamentos $u_{i}(x)$, $1 \leq i \leq 3$. Para o leitor ter uma ideia da sua complexidade escrevem-se as equações por extenso. As equações correspondentes a (23) têm a forma:

$$
\begin{aligned}
& -\frac{\partial \sigma_{11}}{\partial x_{1}}-\frac{\partial \sigma_{12}}{\partial x_{2}}-\frac{\partial \sigma_{13}}{\partial x_{3}}=f_{1} \\
& -\frac{\partial \sigma_{21}}{\partial x_{1}}-\frac{\partial \sigma_{22}}{\partial x_{2}}-\frac{\partial \sigma_{23}}{\partial x_{3}}=f_{2} \\
& -\frac{\partial \sigma_{31}}{\partial x_{1}}-\frac{\partial \sigma_{32}}{\partial x_{2}}-\frac{\partial \sigma_{33}}{\partial x_{3}}=f_{3}
\end{aligned}
$$

e as correspondentes a (25):

$$
\begin{aligned}
& \sigma_{11} n_{1}+\sigma_{12} n_{2}+\sigma_{13} n_{3}=F_{1}, \\
& \sigma_{21} n_{1}+\sigma_{22} n_{2}+\sigma_{23} n_{3}=F_{2}, \\
& \sigma_{31} n_{1}+\sigma_{32} n_{2}+\sigma_{33} n_{3}=F_{3},
\end{aligned}
$$

onde se deve substituir a lei de Hooke generalizada (22). No caso unidimensional estas equações reduzem-se a

$$
\begin{gathered}
\left.-\left(E^{H} A u^{\prime}\right)^{\prime}=f=0, \quad \text { em }\right] 0, L[, \\
u(0)=0, \\
\left(E^{H} A u^{\prime}\right)=F, \text { no extremo } x=L,
\end{gathered}
$$

respectivamente, cuja formulação variacional é dada por (4). Neste mesmo caso, a lei de Hooke tomou a forma particular seguinte:

$$
\sigma_{11}=E^{H} \quad e_{11}(u)=E^{H} u^{\prime},
$$

onde se considerou que a direcção axial da barra coincidia com a do eixo das abcissas $O x_{1}$.

Assim, no caso tridimensional, o funcional a minimizar toma a forma:

$$
\int_{\Omega} f_{i} u_{i} d x+\int_{\Gamma_{i}} F_{i} u_{i} d s+k \int_{\Omega} \rho^{H},
$$


sujeito à equação de estado seguinte;

$$
\int_{\Omega} E_{i j k m n}^{H} e_{k m m}(u) e_{i j}(v) d x=\int_{\Omega} f_{i} v_{i} d x+\int_{\Gamma_{1}} F_{i} v_{i} d s, \quad \forall v \in V,
$$

onde agora $V=\left\{v \in\left[H^{1}(\Omega)\right]^{3}: v=0\right.$ sobre $\left.\Gamma_{0}\right\}$, e que não é mais do que a versão variacional das equações de equilibrio (23)-(25).

$\mathrm{O}$ lagrangiano toma agora a forma:

$$
\begin{aligned}
L= & \int_{\Omega} f_{i} u_{i} d x+\int_{\Gamma_{1}} F_{i} u_{i} d s+k \int_{\Omega} \rho^{H}+ \\
& +\lambda\left[\int_{\Omega} E_{i j k m}^{H} e_{k m}(u) e_{i j}(v) d x=\int_{\Omega} f_{i} v_{i} d x+\int_{\Gamma_{1}} F_{i} v_{i} d s\right]+ \\
& +\int_{\Omega} \tau^{+}(\gamma-1) d x+\int_{\Omega} \tau^{-} \gamma d x,
\end{aligned}
$$

Das condições necessárias de estacionaridade obtêm-se as equações (6), (8)-(11), (37), sendo a equação (7) substituída por:

$$
k \frac{d \rho^{H}}{d \gamma}-\frac{d E_{i j k m}^{H}}{d \gamma} e_{k m}(u) e_{i j}(u)+\tau^{+}-\tau^{-}=0,
$$

Torna-se agora necessário calcular as derivadas em ordem à fracção volúmica $\gamma$ da densidade e coeficientes de elasticidade homogeneizados, $\rho^{H}$ e $E_{i j k m}^{H}$, respectivamente. Para laminados simples, formados por materiais com propriedades elásticas $E_{i j k m}^{+}$e $E_{i j k m}^{-}$, nas proporções $\gamma \mathrm{e}$ $1-\gamma$ respectivamente, tem-se, para o caso bidimensional:

$$
\begin{aligned}
E_{1111}^{H}= & \frac{E_{1111}^{+} E_{1111}^{-}}{\gamma E_{1111}^{-}+(1-\gamma) E_{1111}^{+}}, \\
E_{1212}^{H}= & \frac{E_{1212}^{+} E_{1212}^{-}}{\gamma E_{1212}^{-}+(1-\gamma) E_{1212}^{+}}, \\
E_{1122}^{H}= & {\left[\gamma \frac{E_{1122}^{+}}{E_{1111}^{+}}+(1-\gamma) \frac{E_{1122}^{-}}{E_{1111}^{-}}\right] \frac{E_{1111}^{+} E_{1111}^{-}}{\gamma E_{1111}^{-}+(1-\gamma) E_{1111}^{+}}, } \\
E_{2222}^{H}= & \gamma E_{2222}^{+}+(1-\gamma) E_{2222}^{-}-\left[\gamma \frac{\left(E_{1122}^{+}\right)^{2}}{E_{1111}^{+}}+(1-\gamma) \frac{\left(E_{1122}^{-}\right)^{2}}{E_{1111}^{-}}\right]+ \\
& +\left[\gamma \frac{E_{1122}^{+}}{E_{1111}^{+}}+(1-\gamma) \frac{E_{1122}^{-}}{E_{1111}^{-}}\right]^{2} \frac{E_{1111}^{+} E_{1111}^{-}}{\gamma E_{1111}^{-}+(1-\gamma) E_{1111}^{+}} .
\end{aligned}
$$


No caso de tensão plana, estas expressões simplificam-se e tem-se:

$$
\begin{aligned}
& E_{1111}^{H}=\frac{1}{1-v^{2}} I_{1}, \\
& E_{1212}^{H}=\frac{1}{2(1+v)} I_{1}, \\
& E_{1122}^{H}=\frac{v}{1-v^{2}} I_{1}, \\
& E_{2222}^{H}=I_{2} \frac{v^{2}}{1-v^{2}} I_{1},
\end{aligned}
$$

onde

$$
I_{1}=\frac{E^{+} E^{-}}{\gamma E^{-}+(1-\gamma) E^{+}}, \quad I_{2}=\gamma E^{+}+(1-\gamma) E^{-},
$$

e onde $v$ designa o coeficiente de Poisson, que se supõe o mesmo para ambos os materiais. Trata-se da generalização das equações (12), onde $\rho^{H}$ mantém a mesma forma.

A dependência destas expressões em $\gamma$ é simples, pelo que as derivadas em (39) são de cálculo imediato. No caso de se optar por uma representação da microestrutura mais complexa, permitindo, por exemplo, a rotação da microestrutura, o que se torna necessário para análise do fémur, há que recorrer às técnicas de derivação numérica, mas as ideias fundamentais são as expostas ( $c f$. Bendsøe, M. P., e Kikuchi, N., 1988; Bendsøe, M. P., 1995; Murat, F., e Tartar, L., 1997; e Fernandes, P. R., Folgado, J., e Miranda, P. S., 2000).

\section{EXEMPLOS NUMÉRICOS APLICADOS À ANÁLISE DA ESTRUTURA ÓSSEA DO FÉMUR}

Utilizando o método dos elementos finitos para a resolução numérica do problema da elasticidade e um algoritmo de optimização para resolver a condição necessária de estacionaridade (39), Fernandes, P., Rodrigues, H., e Jacobs, C., (1999) obtiveram o resultado ilustrado na figura seguinte: 

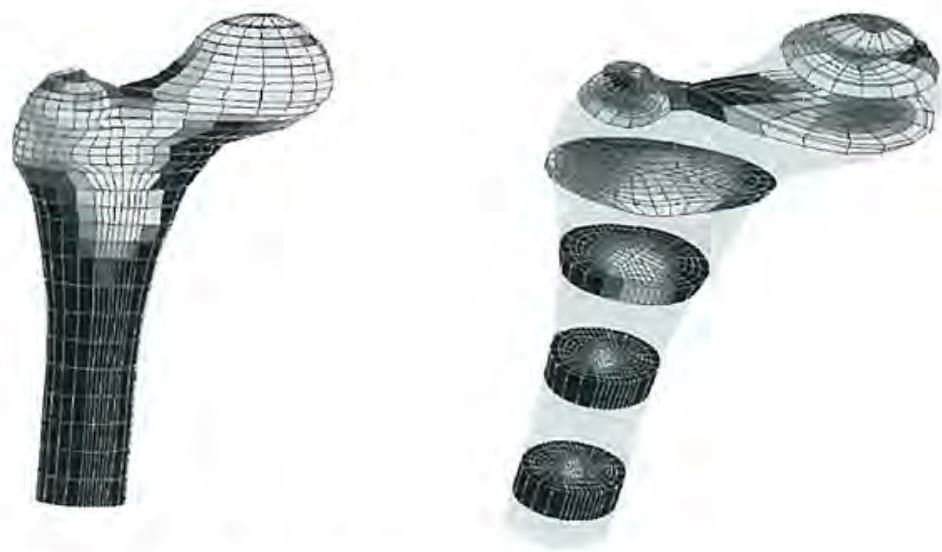

Fig. 4-Distribuição de densidade no fémur

A comparação da distribuição, macroscópica, da densidade com uma radiografia está feita na figura seguinte:
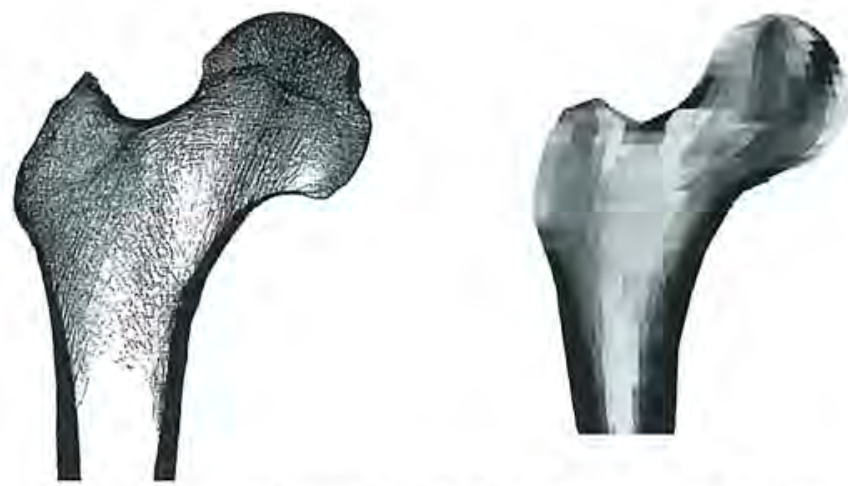

Fig. 5 - Comparação entre o resultado numérico e uma radiografia

Calculando em cada ponto os valores e vectores próprios do tensor das tensões de Cauchy, $\sigma_{i j}$, obtêm-se os valores e as direcções de maior tensão mecânica. Representando estas grandezas graficamente, observa-se uma boa correlação com a lei de Wolff, notando-se nitidamente, o desenvolvimento do osso trabecular ao longo das direcções (vectores 
próprios) de tensões mais elevadas (valores próprios) do tensor das tensões de Cauchy. Este aspecto teórico e numérico da lei de Wolff encontra-se representado na figura seguinte:

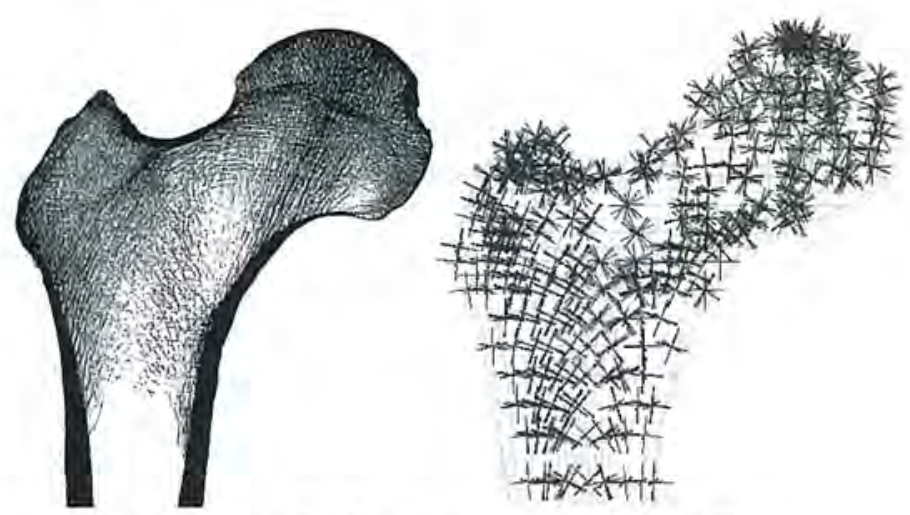

Fig. 6-Verificaçāo numérica da lei de Wolff

Possuindo um algoritmo com estas características, nada impede que se faça o estudo simultâneo do conjunto osso-prótese. O resultado numérico obtido por Fernandes, Folgado e Miranda, 2000, encontra-se representado na figura seguinte:
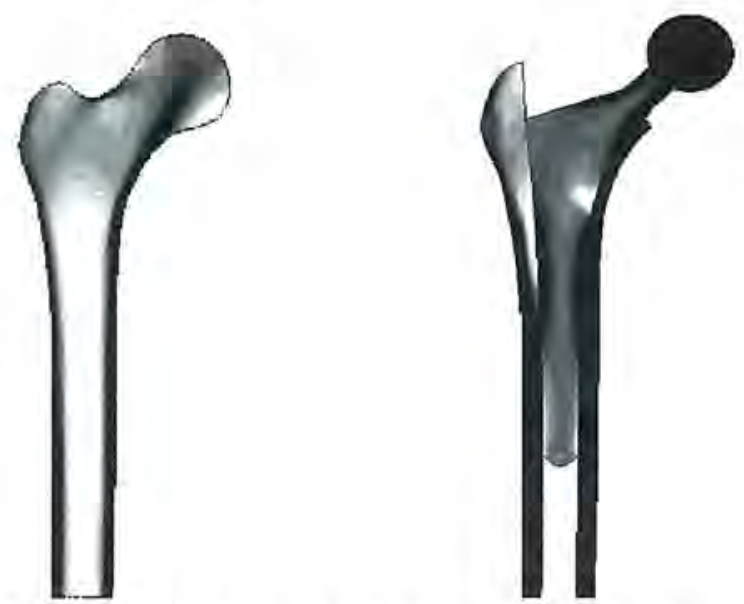

Fig. 7-Distribuição numérica da densidade com e sem prótese 
Resultado este de grande utilidade no projecto e desenvolvimento de próteses, bem como no eventual acompanhamento, no futuro, da prática clínica.

\section{Referências}

Bendsøe, M. P., e N. Kikuchi (1988) - Generating optimal topologies in structural design using a homogenization method, Comp. Meth. Appl. Mech. Engng., $71,192-224$.

Bendsøe, M. P., (1995) - Optimization of structural topology, shape, and material, Berlim, Springer Verlag.

Guedes, J. M., e N. Kikuchi (1990) - preprocessing and postprocessing for materials based on the homogenization method with adaptive finite element methods, Comp. Meth. Appl. Sci. Engnr., 83, 143-198.

Murat, F., e L. Tartar (1997) - consultar os vários artigos em Topics in the Mathematical Modelling of Composite Materials, A. Cherkaev e B. Khon, eds.; Birhäuser.

P. Fernandes, H. Rodrigues e C. Jacobs (1999) - A Model of Bone Adaptation Using a Global Optimisation Criterion Based On the Trajectorial Theory of Wolff; Computer Methods in Biomechanics and Biomedical Engineering, vol, 2, p. 125-138.

P. R, Fernandes, J. Folgado e P. S. Miranda (2000) - Modelos Computacionais para o Projecto de Próteses Ortopédicas, VI Congresso Nacional de Mecânica Aplicada e Computacional, vol. 1, pp. 135-144. 
(Página deixada propositadamente em branco) 


\section{$\begin{array}{llllll}\text { C I E } & \mathbf{N} & \mathbf{C} & \mathbf{A}\end{array}$}

mitiram realizar com sucesso tarefas tão distintas como a programação de um voo a Marte, a previsão de resultados eleitorais, a explicação do funcionamento de alguns mecanismos do sistema nervoso, ou a abordagem critica de obras de arte e de textos literários. Da ciência à sociedade, dos grandes avanços técnicos à solidez de uma argumentação lógica, a Matemática constrói teias de uma imensa flexibilidade resultante do carácter universal da sua linguagem.

Neste livro, personalidades de diferentes universos dão o seu testemunho sobre a forma como usam as teias matemáticas para tecer a sua própria visão do mundo.

Maria Paula Serra de Oliveira é professora de Matemática na Faculdade de Ciências e Tecnologia da Universidade de Coimbra. 